\title{
Anti-Stokes Raman Scattering of Single Carbyne Chains
}

\author{
Cla Duri Tschannen,* Martin Frimmer, Georgy Gordeev, Thiago L. Vasconcelos, Lei Shi, \\ Thomas Pichler, Stephanie Reich, Sebastian Heeg, and Lukas Novotny
}

Cite This: ACS Nano 2021, 15, 12249-12255

Read Online

ABSTRACT: We investigate the anti-Stokes Raman scattering of single carbyne chains confined inside double-walled carbon nanotubes. Individual chains are identified using tip-enhanced Raman scattering (TERS) and heated by resonant excitation with varying laser powers. We study the temperature dependence of carbyne's Raman spectrum and quantify the laser-induced heating based on the anti-Stokes/Stokes ratio. Due to its molecular size and its large Raman cross section, carbyne holds great promise for local temperature monitoring, with potential applications ranging from nanoelectronics to biology.
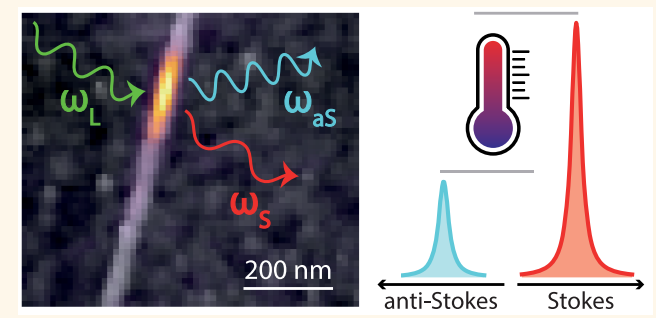

KEYWORDS: carbyne, linear carbon chains, Raman scattering, TERS, Raman thermometry, anti-Stokes, nanometrology

\section{INTRODUCTION}

Carbyne, the paradigmatic sp-hybridized and truly onedimensional allotrope of carbon, ${ }^{1,2}$ has attracted significant interest due to its anticipated outstanding mechanical, ${ }^{3}$ thermal, ${ }^{4}$ and electronic ${ }^{5}$ properties. In particular, carbyne possesses a band gap that is sensitive to external perturbations $^{6-10}$ and can possibly be switched externally from a semiconducting to a metallic state, ${ }^{11,12}$ which points out the potential of carbyne for nanoelectronic devices. While the synthesis of carbyne has long been challenging, ${ }^{13-15}$ large progress has been made in recent years using carbon nanotubes as nanoreactors and protective nanocontainers, ${ }^{16-23}$ as illustrated in Figure 1a.

As for other nanomaterials, Raman spectroscopy has played an important role in the investigation of carbyne, ${ }^{6-8,17,19}$ owing to its sensitivity to structural, electronic, optical, and chemical information. ${ }^{24-27}$ At the heart of Raman spectroscopy lies the inelastic scattering of light by quantized lattice vibrations (phonons). The anti-Stokes process, being related to the annihilation of a phonon, can only occur if a phonon is already present in the system. The anti-Stokes scattering intensity is therefore proportional to the phonon population $n$. Stokes scattering, on the other hand, can also occur in the absence of a phonon. As a result, the Stokes Raman scattering intensity is proportional to $n+1 . \cdot^{25,28}$ For phonons with an energy larger than the thermal energy, phonon population numbers are very small $(n \ll 1)$. Consequently, at room temperature, anti-Stokes signals of high-energy $\left(<1000 \mathrm{~cm}^{-1}\right)$ Raman modes are several orders of magnitude weaker than their Stokes counterparts. This discrepancy explains why for nanoscale systems, anti-Stokes spectra of high-energy Raman modes are typically only accessible for bulk quantities, ${ }^{29}$ in solution, ${ }^{30}$ or under exotic conditions such as surface-enhanced Raman scattering, ${ }^{31}$ bias-induced hot phonon generation, ${ }^{32}$ or correlated Stokes-anti-Stokes scattering. ${ }^{33}$

For carbyne, however, the situation looks different. As recently demonstrated, carbyne has a resonant Raman scattering cross section per atom exceeding that of any other known material or molecule by at least two orders of magnitude. $^{34}$ This record-high Raman cross section suggests that anti-Stokes spectroscopy of isolated carbyne chains is possible without additional enhancement, despite the low occupation number associated with carbyne's high-energy $\mathrm{C}$ mode optical phonon ( $n \approx 1.4 \times 10^{-4}$ at room temperature). Yet, the Raman spectroscopic characterization of carbyne has so far been restricted to Stokes scattering. ${ }^{6-8,17,19}$ This is surprising, for extending the scope to the anti-Stokes side of the spectrum potentially provides a means for temperature sensing on the nanoscale. In addition, anti-Stokes measurements can aid in assessing fundamental material properties such as phonon lifetimes ${ }^{35}$ and optical transition energies, ${ }^{36}$ as well as extending the mechanistic understanding of the Raman process. $^{30}$

Received: May 7, 2021

Accepted: June 25, 2021

Published: July 13, 2021 
(a)
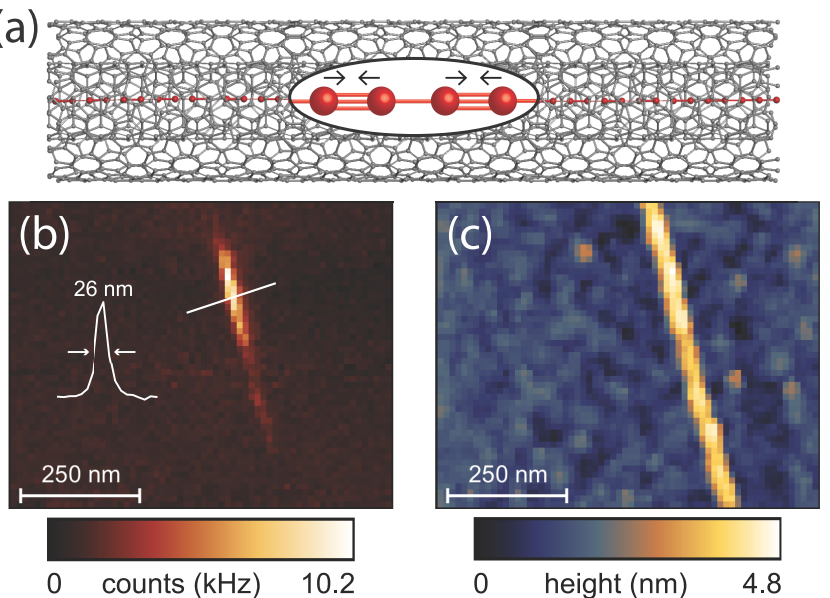

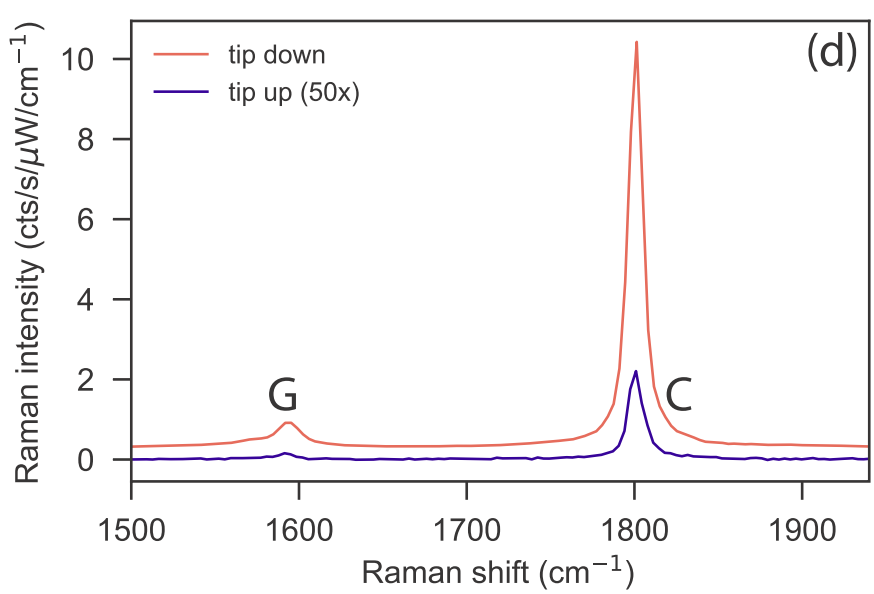

Figure 1. Tip-enhanced Raman scattering of confined carbyne. (a) Carbyne chain encapsulated in a DWCNT. The inset illustrates the atomic structure of carbyne, which is characterized by alternating single and triple bonds. Arrows illustrate the Raman-active vibrational C-mode. (b, c) Simultaneously acquired optical (b) and topographic (c) image of a single confined carbyne chain. The intensity profile in (b) extracted along the white line indicates a spatial resolution of $26 \mathrm{~nm}$. (d) Near-field (tip down) and far-field (tip up) Raman spectrum of confined carbyne. The strong C-peak at $1801 \mathrm{~cm}^{-1}$ is associated with the carbyne chain, whereas the G-peak signature below $1801 \mathrm{~cm}^{-1}$ arises from the encapsulating DWCNT structure. Note that the far-field spectrum is scaled by a factor of 50 for better visibility.

In this work, we demonstrate anti-Stokes Raman scattering of single carbyne chains. Using laser irradiation as a heating source, we investigate the temperature dependence of the Cmode Stokes and anti-Stokes peaks. We show that the evolution of the anti-Stokes/Stokes ratio with laser power is well captured by the Boltzmann factor with a power-dependent equilibrium temperature, allowing us to quantify the laserinduced heating. The potential of carbyne as a nanoscale temperature sensor is discussed and possible applications are outlined.

\section{RESULTS AND DISCUSSION}

Synthesis and Near-Field Raman Spectroscopy of Confined Carbyne. Carbyne chains are synthesized inside double-walled carbon nanotubes (DWCNTs) by high-temperature annealing according to the procedure described by Shi et al. $^{20}$ The tubes are then dispersed on a thin glass coverslip following ref 7 .

Our work makes use of both near-field and far-field measurements, each carried out on a separate Raman setup. The first step is to identify an isolated, continuous carbyne chain confined in a DWCNT. To this end, we make use of the nanoscale resolution provided by tip-enhanced Raman scattering (TERS). The TERS measurements are performed in a backscattering configuration using a home-built setup (see Methods). As an optical probe we use a plasmon-tunable tip pyramid designed to support localized surface plasmon resonances at the excitation energy of our laser $(1.96 \mathrm{eV})$, which gives rise to particularly strong Raman scattering enhancement. ${ }^{37-39}$ Raman scattered photons are sent through a narrow band-pass filter that transmits only the spectral region of carbyne's C-mode and then detected with an avalanche photodiode (APD). Optical and topographic images are formed simultaneously by raster-scanning (see Figure 1b,c). Full spectra are recorded using a CCD-equipped spectrometer (Figure 1d).

Power-Dependent Resonant Far-Field Raman Spectroscopy. In the second experimental step, we perform power-dependent far-field Raman measurements (see Methods) of the previously identified carbyne chain. To profit from resonant Raman scattering enhancement, we tune the photon energy of our laser to coincide with the band gap of the investigated carbyne chain $(1.88 \mathrm{eV})$. We infer the band gap energy by exploiting its linear scaling with the measured $\mathrm{C}$ mode Raman shift $\left(1801 \mathrm{~cm}^{-1}\right.$ according to Figure $\left.1 \mathrm{~d}\right){ }^{6-8} \mathrm{We}$ point out that the band gap energy and Raman peak position of confined carbyne are both affected by the noncovalent interaction with the encasing host nanotube, which in turn depends on the nanotube chirality. Therefore, carbyne chains encapsulated in nanotubes of different chirality differ in their vibrational and electronic properties. $^{6-8}$

Figure 2 shows our power-dependent far-field Stokes and anti-Stokes measurements. A series of anti-Stokes spectra, measured at five different values of laser power, are displayed in Figure 2a. We fit each spectrum with a Lorentzian line shape on top of a linear background. In Figure $2 b$ we display the corresponding series of Stokes spectra. We find equal Raman shifts for the Stokes and anti-Stokes peaks, as expected for a first-order single-resonance Raman process. ${ }^{25,28}$ Moreover, we observe a softening of the C-mode Raman shift with increasing laser power. Such frequency downshifts have been observed in temperature- and power-dependent Raman studies of a wide variety of materials ${ }^{40-44}$ and can be attributed to anharmonic terms of the lattice potential energy. ${ }^{25}$ We provide a brief discussion of this effect in the Supporting Information.

To shed light on the power dependence of the Raman signals, we extract the integrated C-peak anti-Stokes intensities $A_{\mathrm{C}}^{\mathrm{aS}}$ from the fits in Figure $2 \mathrm{a}$, and the Stokes intensities $A_{\mathrm{C}}^{\mathrm{S}}$ from the fits in Figure $2 \mathrm{~b}$. We plot these Stokes and anti-Stokes signals in Figure $2 c$ as a function of excitation power. To illustrate the power-dependent behavior of the Stokes and antiStokes signals, we fit both sets of data with a second-order polynomial, plotted in Figure $2 \mathrm{c}$ as solid lines. Inspection of Figure $2 c$ demonstrates that the anti-Stokes and Stokes Raman signals show opposing trends with increasing excitation power. The anti-Stokes signal $A_{\mathrm{C}}^{\mathrm{aS}}$ increases supra-linearly, which can be ascribed to the temperature dependence of the C-mode phonon population given by the Bose-Einstein distribution, $n$ $=\left\{\exp \left[E_{\mathrm{ph}} /\left(k_{\mathrm{B}} T\right)\right]-1\right\}^{-1}$, where $E_{\mathrm{ph}}$ is the phonon energy, $k_{\mathrm{B}}$ is the Boltzmann constant, and $T$ is the temperature. Laser- 

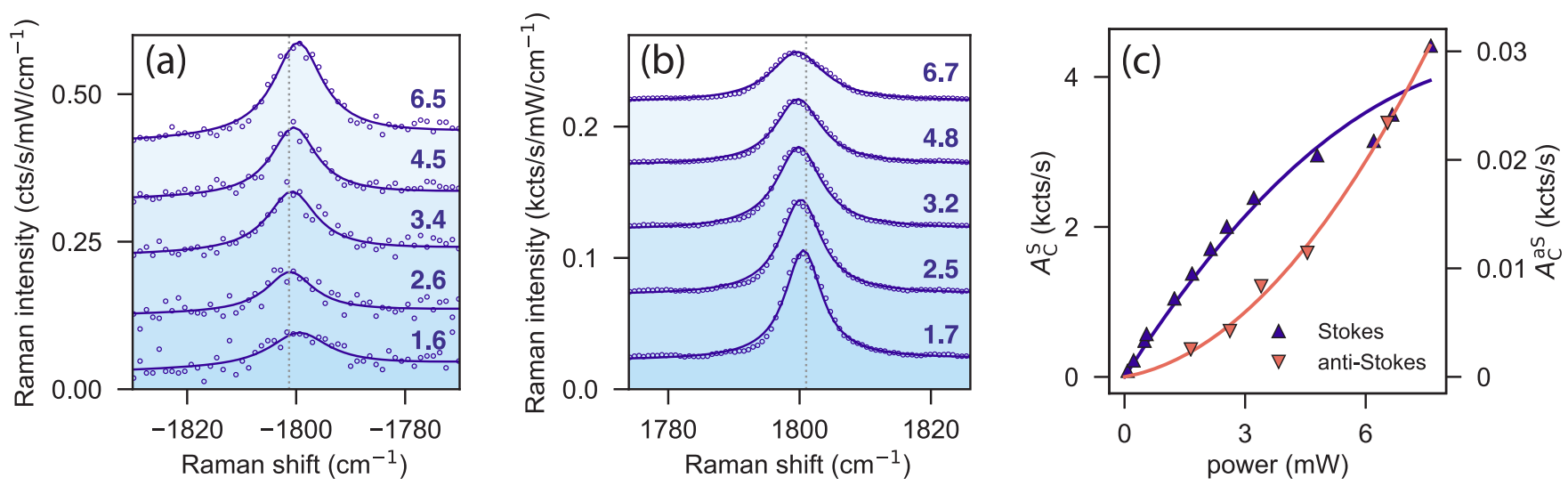

Figure 2. Power-dependent anti-Stokes and Stokes Raman spectroscopy of an individual confined carbyne chain. (a) Anti-Stokes and (b) Stokes spectra recorded at different excitation powers, which are indicated in blue in units of $\mathrm{mW}$. The spectra are normalized by the excitation power and offset vertically for better visibility. The lines are Lorentzian fits on a linear background. The gray dotted lines at $\pm 1801 \mathrm{~cm}^{-1}$ serve as reference to illustrate changes in the peak position. (c) Stokes $\left(A_{\mathrm{C}}^{\mathrm{S}}\right)$ and anti-Stokes $\left(A_{\mathrm{C}}^{\mathrm{aS}}\right)$ Raman signals as a function of excitation power. The lines are second-order polynomial fits.

induced heating increases the phonon occupation number $n$, which in turn gives rise to stronger anti-Stokes scattering. The Stokes signal $A_{\mathrm{C}}^{\mathrm{S}}$, on the other hand, grows sublinearly with laser power. We attribute this behavior to changes of the underlying Raman resonance due to laser heating. These changes can include a shift of the optical transition energy as well as an enhanced nonradiative damping of the photoexcited state. Models including such effects have been frequently employed in power- and temperature-dependent Raman studies of low-dimensional semiconductors. ${ }^{45-47}$ Moreover, this explanation is in line with the strong C-mode Raman intensity increase observed by Shi et al. at cryogenic temperatures in bulk measurements. ${ }^{20}$ We emphasize that while the exact mechanism behind the saturation of the Stokes Raman signal toward high excitation powers in Figure $2 \mathrm{c}$ requires further investigation, it has no relevant effect on the anti-Stokes/Stokes ratio. This robustness of the anti-Stokes/ Stokes ratio of carbyne against changes of the electronic structure is a consequence of the large $\mathrm{C}$-mode phonon energy, as elaborated in the final part of this section.

After having discussed the power-dependence of the antiStokes and Stokes signals separately, we now focus on their ratio $A_{\mathrm{C}}^{\mathrm{aS}} / A_{\mathrm{C}}^{\mathrm{S}}$ and its power dependence. In Figure 3 we plot the ratio of the two polynomial fits from Figure $2 c$ as a solid line. This curve represents our experimental anti-Stokes/Stokes ratio as a function of laser power. Note that going from Figure $2 \mathrm{c}$ to Figure 3, we have applied corrections of the anti-Stokes/ Stokes ratio for the $\omega^{4}$ dependence of Raman scattering $25,28,48$ as well as for the frequency dependence of the instrument. Details on the calibration procedure are given in the Supporting Information.

With this calibration, we expect the anti-Stokes/Stokes ratio to match the Boltzmann factor $A_{\mathrm{C}}^{\mathrm{aS}} / A_{\mathrm{C}}^{\mathrm{S}}=\exp \left[-E_{\mathrm{ph}} /\left(k_{\mathrm{B}} T\right)\right]^{25,28}$ Indeed, as shown in Figure 3, we find that laser irradiation increases the anti-Stokes/Stokes ratio and the associated phonon equilibrium temperature $T$. Assuming that the temperature scales linearly with excitation power $P_{\mathrm{L}}$, we can extract the rate $C_{\mathrm{T}}$ of laser-induced heating from fitting a modified Boltzmann factor, $B \exp \left\{-E_{\mathrm{ph}} /\left[k_{\mathrm{B}}\left(293+C_{\mathrm{T}} P_{\mathrm{L}}\right)\right]\right\}$. The fit is shown as the red dashed line in Figure 3 and yields a heating rate of $C_{\mathrm{T}}=11.1(+2.4 /-1.8) \mathrm{K} / \mathrm{mW}$. We elaborate on the accuracy of this estimate in the Supporting Information. The free parameter $B$, for which we obtain a value of 1.29 ,

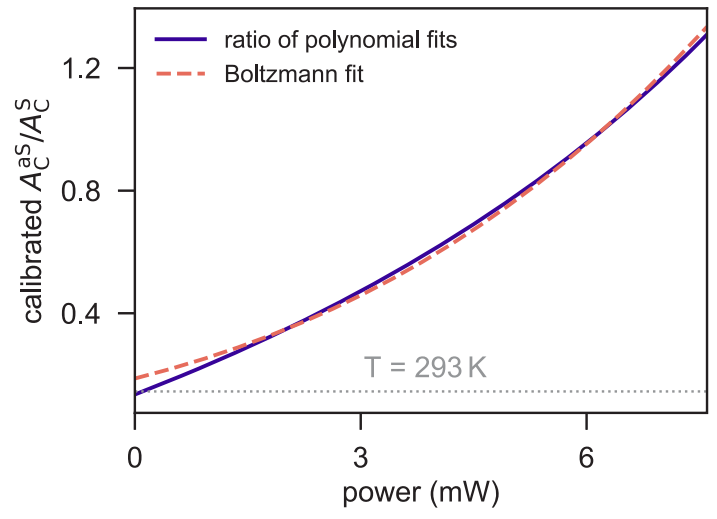

Figure 3. Anti-Stokes/Stokes ratio $\left(A_{\mathrm{C}}^{\mathrm{aS}} / A_{\mathrm{C}}^{\mathrm{S}}\right)$ of a single confined carbyne chain as a function of excitation power. The blue line is the ratio of the two polynomial fits shown in Figure $2 \mathrm{c}$. The red dashed line is a fit to the blue curve of the Boltzmann factor with a power-dependent equilibrium temperature, as explained in the main text. The gray dotted line represents the Boltzmann factor at room temperature $(293 \mathrm{~K})$.

accounts for uncertainties of the calibration procedure. When extrapolated to zero power, both the anti-Stokes/Stokes ratio and the Boltzmann fit in Figure 3 closely coincide with the room-temperature Boltzmann factor (gray dotted line). This finding supports the accuracy of our calibration and corroborates that we excite the carbyne chain near its electronic resonance. ${ }^{7,29,49}$ Moreover, from the agreement of the two curves in Figure 3, we conclude that heating is the main contribution to the change of the anti-Stokes/Stokes ratio as the laser power is increased. Thus, correlated Stokesanti-Stokes scattering (a nonlinear process) does not play a sizable role, ${ }^{33,50}$ as we further expand upon in the Supporting Information. Based on our estimate of the heating rate $C_{\mathrm{T}}$, the power range depicted in Figure 3 corresponds to a temperature increase of roughly $90 \mathrm{~K}$, which leads to the observed increase of the anti-Stokes/Stokes ratio by one order of magnitude. As a consistency check for our results, we reproduce them in the Supporting Information Figures $2 \mathrm{c}$ and 3 with another carbyne chain of similar C-mode frequency and find good agreement between the two carbyne chains. 
Potential of Carbyne for Nanoscale Thermometry. Let us now discuss the prospects of single carbyne chains as optically interrogated temperature probes of molecular dimension. In our work, we use resonant Raman scattering, that is, our excitation frequency is resonant with the carbyne band gap. As discussed above, we argue that the temperature dependence of this resonance with respect to energetic position and width explains the sublinear dependence of the Stokes Raman signal on excitation power (see Figure 2c). Importantly, a change in resonance influences the Stokes and anti-Stokes signals of carbyne to the same extent, thus rendering their ratio immune to changes of the resonance's position and width. This robustness persists as long as the resonance shift remains small compared to the phonon energy. In this regime, only the resonance condition for the excitation laser plays a role (incoming resonance). ${ }^{7}$ On the other hand, too large of a temperature-induced resonance shift will bring the Stokes or anti-Stokes emission into resonance (outgoing resonance). In that regime, the anti-Stokes/Stokes ratio starts to deviate from the Boltzmann factor, ${ }^{29,49}$ which complicates the extraction of a temperature.

From our measurements, we extract a heating-induced shift of the C-mode Raman peak of around $-2 \mathrm{~cm}^{-1}$ (see Figure $2 b$ and Supporting Information), corresponding to a thermal shift of the band gap energy of $-13 \mathrm{meV}$. ${ }^{6,8} \mathrm{We}$ underline that the direct correlation between Raman frequency and optical transition energy is an inherent property of confined carbyne $^{6,7,15}$ that sets carbyne apart from other nanomaterials for which the influence of temperature on electronic structure is more difficult to assess. ${ }^{45-47,49,51,52}$ Given the much larger C-mode phonon energy of more than $220 \mathrm{meV}$, the measured temperature-induced downshift of the band gap by $13 \mathrm{meV}$ has no relevant effect on the anti-Stokes/Stokes ratio of carbyne. Hence, in the context of Raman thermometry, large phonon energies allow for reliable and robust temperature extraction from the anti-Stokes/Stokes ratio, regardless of changes of the electronic structure due to temperature variations. While antiStokes signals of high-energy phonons are in general challening to obtain due to low phonon occupation numbers, carbyne overcomes this difficulty by virtue of its record-high Raman scattering cross-section, ${ }^{34}$ allowing for anti-Stokes measurements - and thus, potentially, robust thermometry-at the single chain level.

Finally, let us briefly balance the potential of carbyne for local temperature sensing against other Raman-based methods. For instance, organic dye molecules have been used for nanoscale temperature sensing in the context of surfaceenhanced Raman scattering. ${ }^{31,53}$ However, without additional plasmonic enhancement the Raman scattering cross section of such molecules is much lower than that of carbyne. ${ }^{34}$ In addition, organic dyes are very sensitive to photodegradation, while we demonstrate here that carbyne survives few-milliwatts continuous wave illumination under a $100 \times$ objective for prolonged integration times. Carbon nanotubes are another nanoscale system whose anti-Stokes/Stokes ratio has been studied, in particular of the radial-breathing mode (RBM). ${ }^{29,36,49}$ The RBM is a low-energy phonon and has a comparatively large Raman scattering cross section. ${ }^{54}$ From a phonon population point of view, it might appear promising to use the RBM for anti-Stokes thermometry, due to larger roomtemperature occupation numbers compared to the C-mode of carbyne. On the downside, anti-Stokes thermometry using lowenergy phonons faces the problem of an unknown evolution of the electronic structure with temperature described above. We point out that knowledge about the electronic structure is only required for resonant excitation, but nonresonant excitation is not an option because the associated Raman signals are generally weaker by orders of magnitude. Lastly, Raman thermometry can also be performed on the basis of temperature-dependent features other than the anti-Stokes/ Stokes ratio, such as the frequently observed mode softening with increasing tempeature. ${ }^{40,41,43,55}$ However, compared to Raman thermometry using temperature-induced peak shifts, the anti-Stokes/Stokes ratio has the key benefit of being selfreferencing. That is, while absolute peak positions of lowdimensional materials can be affected by the local environment due to stress or doping, ${ }^{26,56-58}$ the anti-Stokes/Stokes ratio always reports the absolute local temperature.

In summary, carbyne stands out compared to other molecular systems because of its distinctive combination of large phonon energy, ${ }^{15,59}$ record-high Raman scattering cross section, ${ }^{34}$ and transparent evolution of the band gap energy with temperature. $^{6-8}$ Therefore, carbyne holds great promise for anti-Stokes Raman thermometry on the nanoscale.

\section{CONCLUSION AND OUTLOOK}

In conclusion, we have experimentally shown that the unparalleled Raman scattering cross section of carbyne enables anti-Stokes spectroscopy at the single chain level. Using laser irradiation as a heating source, we have investigated the temperature dependence of the Stokes and anti-Stokes Raman peaks. Finally, we have demonstrated that the anti-Stokes/ Stokes ratio of carbyne offers untapped potential for local temperature sensing. Our work is an important step toward a practical method for all-optical probing of temperature variations on the nanoscale, with possible applications in many areas of modern science and technology.

For instance, carbyne-based Raman thermometry offers a promising perspective for thermal management on the length scale of emerging ultracompact device architectures, which is a key requirement for their functioning and control. ${ }^{60-62}$ This prospect bears particular relevance in light of the recent progress in the synthesis of carbyne chains with tailored properties, which puts the integration of carbyne into the next generation of nanoelectronic devices firmly within reach. ${ }^{23,63,64}$

The integration of carbyne within a living system may offer further intriguing possibilities, ranging from tuning gene expression by simultaneously monitoring and controlling subcellular thermal gradients ${ }^{65-67}$ to investigating local tumor activity by mapping atypical thermogenesis at the single-cell level. ${ }^{68}$ On top, the demonstrated robustness of carbyne toward large-power continuous-wave laser irradiation for prolonged integration times adds to the list of advantages that would make it a powerful marker for use in biological imaging. ${ }^{34}$

Finally, our measurements constitute a starting point for the further investigation of anti-Stokes scattering in carbyne. In particular, exploring the outgoing anti-Stokes resonance might not only increase the absolute anti-Stokes signal, as shown for carbon nanotubes, ${ }^{30}$ but also shed more light on the exact nature of optical transitions in carbyne. ${ }^{8,24,69}$

\section{METHODS}

Near-Field Raman Measurements. Our TERS setup is based on an inverted optical microscope with a home-built scanning probe microscope on top. The sample coverslip is placed onto an $x-y$ piezo 
scan-stage and illuminated from below with a radially polarized laser beam that is tightly focused by an oil immersion objective (NA 1.4). The TERS probe is attached to a quartz tuning fork and its distance from the sample surface is controlled by a shear-force feedback system. Positioning the probe into the laser focus generates a localized excitation source for Raman scattering. A more detailed description of our TERS system is given in refs 70 and 71 .

Far-Field Raman Measurements. Far-field measurements are carried out in a backscattering geometry with a $100 \times$ air objective (NA 0.9). We align both the polarization of the excitation laser and the direction of polarized detection with the chain's main axis, as detailed in ref 7. A tunable dye laser serves as excitation source. For all power-dependent far-field measurements, we continuously monitor the power of the excitation laser during signal acquisition. All power values reported in this work refer to mean values (averaged over the integration time) as measured before the back aperture of the objective. Also, instead of linearly varying the laser power, we alternate between higher and lower power values. This procedure counteracts any systematic effects introduced by possible drifts of the sample with respect to the laser focus. The integration time for all Stokes (anti-Stokes) measurements is $60 \mathrm{~s}(180 \mathrm{~s})$.

\section{ASSOCIATED CONTENT}

\section{(s) Supporting Information}

The Supporting Information is available free of charge at https://pubs.acs.org/doi/10.1021/acsnano.1c03893.

Power dependence of the Raman spectrum of confined carbyne; uncertainty of the heating rate estimate; calibration of the anti-Stokes/Stokes ratio; powerdependent Stokes and anti-Stokes Raman signals of another confined carbyne chain; investigating the influence of correlated Stokes-anti-Stokes scattering (PDF)

\section{AUTHOR INFORMATION}

\section{Corresponding Author}

Cla Duri Tschannen - Photonics Laboratory, ETH Zürich, 8093 Zürich, Switzerland; 이이.org/0000-0003-1049-

0819; Email: clat@ethz.ch

\section{Authors}

Martin Frimmer - Photonics Laboratory, ETH Zürich, 8093 Zürich, Switzerland

Georgy Gordeev - Department of Physics, Freie Universität Berlin, 14195 Berlin, Germany; 이잉.org/0000-00023273-2105

Thiago L. Vasconcelos - Materials Metrology Division, Instituto Nacional de Metrologia Qualidade e Tecnologia (INMETRO), 25250-020 Duque de Caxias, RJ, Brazil

Lei Shi - School of Materials Science and Engineering, State Key Laboratory of Optoelectronic Materials and Technologies, Nanotechnology Research Center, Guangzhou Key Laboratory of Flexible Electronic Materials and Wearable Devices, Sun Yat-sen University, Guangzhou

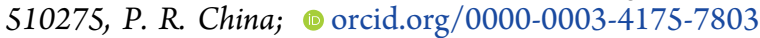

Thomas Pichler - Faculty of Physics, Universität Wien, 1090 Wien, Austria; (1) orcid.org/0000-0001-5377-9896

Stephanie Reich - Department of Physics, Freie Universität

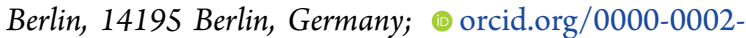
2391-0256

Sebastian Heeg - Department of Physics, Freie Universität Berlin, 14195 Berlin, Germany; Department of Physics, Humboldt Universität zu Berlin, 12489 Berlin, Germany; 으 orcid.org/0000-0002-6485-3083
Lukas Novotny - Photonics Laboratory, ETH Zürich, 8093

Zürich, Switzerland; 이이.org/0000-0002-9970-8345

Complete contact information is available at:

https://pubs.acs.org/10.1021/acsnano.1c03893

\section{Notes}

The authors declare no competing financial interest.

\section{ACKNOWLEDGMENTS}

This work has been supported by the Swiss National Science Foundation (Grant 200020_192362/1). T.L.V acknowledges financial support from $\mathrm{CNPq}$ (305881/2019-1 and 436381/ 2018-4) and MCTI (SibratecNano 21040*16). L.S. acknowledges the financial support from the National Natural Science Foundation of China (Grant 51902353) and Natural Science Foundation of Guangdong Province (Grant 2019A1515011227). S.H. acknowledges financial support by the DFG via Emmy Noether Initiative (HE 8642/1).

\section{REFERENCES}

(1) Heimann, R. B.; Evsyukov, S. E.; Kavan, L. Carbyne and Carbynoid Structures; Springer Science \& Business Media: Dordrecht, 1999.

(2) Hirsch, A. The Era of Carbon Allotropes. Nat. Mater. 2010, 9, 868-871.

(3) Liu, M.; Artyukhov, V. I.; Lee, H.; Xu, F.; Yakobson, B. I. Carbyne from First Principles: Chain of C Atoms, a Nanorod or a Nanorope. ACS Nano 2013, 7, 10075-10082.

(4) Wang, M.; Lin, S. Ballistic Thermal Transport in Carbyne and Cumulene with Micron-Scale Spectral Acoustic Phonon Mean Free Path. Sci. Rep. 2016, 5, 18122.

(5) Tongay, S.; Senger, R. T.; Dag, S.; Ciraci, S. Ab-Initio Electron Transport Calculations of Carbon Based String Structures. Phys. Rev. Lett. 2004, 93, 136404.

(6) Shi, L.; Rohringer, P.; Wanko, M.; Rubio, A.; Waßerroth, S.; Reich, S.; Cambré, S.; Wenseleers, W.; Ayala, P.; Pichler, T. Electronic Band Gaps of Confined Linear Carbon Chains Ranging from Polyyne to Carbyne. Phys. Rev. Mater. 2017, 1, 075601.

(7) Heeg, S.; Shi, L.; Pichler, T.; Novotny, L. Raman Resonance Profile of an Individual Confined Long Linear Carbon Chain. Carbon 2018, 139, 581-585.

(8) Heeg, S.; Shi, L.; Poulikakos, L. V.; Pichler, T.; Novotny, L. Carbon Nanotube Chirality Determines Properties of Encapsulated Linear Carbon Chain. Nano Lett. 2018, 18, 5426-5431.

(9) Yang, X.; Lv, C.; Yao, Z.; Yao, M.; Qin, J.; Li, X.; Shi, L.; Du, M.; Liu, B.; Shan, C.-X. Band-Gap Engineering and Structure Evolution of Confined Long Linear Carbon Chains@Double-Walled Carbon Nanotubes under Pressure. Carbon 2020, 159, 266-272.

(10) Sharma, K.; Costa, N. L.; Kim, Y. A.; Muramatsu, H.; Barbosa Neto, N. M.; Martins, L. G. P.; Kong, J.; Paschoal, A. R.; Araujo, P. T. Anharmonicity and Universal Response of Linear Carbon Chain Mechanical Properties under Hydrostatic Pressure. Phys. Rev. Lett. 2020, 125, 105501.

(11) Rusznyák, A.; Zólyomi, V.; Kürti, J.; Yang, S.; Kertesz, M. Bond-Length Alternation and Charge Transfer in a Linear Carbon Chain Encapsulated within a Single-Walled Carbon Nanotube. Phys. Rev. B: Condens. Matter Mater. Phys. 2005, 72, 155420.

(12) Wanko, M.; Cahangirov, S.; Shi, L.; Rohringer, P.; Lapin, Z. J.; Novotny, L.; Ayala, P.; Pichler, T.; Rubio, A. Polyyne Electronic and Vibrational Properties under Environmental Interactions. Phys. Rev. B: Condens. Matter Mater. Phys. 2016, 94, 195422.

(13) Gibtner, T.; Hampel, F.; Gisselbrecht, J.-P.; Hirsch, A. End-Cap Stabilized Oligoynes: Model Compounds for the Linear sp Carbon Allotrope Carbyne. Chem. - Eur. J. 2002, 8, 408-432. 
(14) Chalifoux, W. A.; Tykwinski, R. R. Synthesis of Polyynes to Model the sp-Carbon Allotrope Carbyne. Nat. Chem. 2010, 2, 967971.

(15) Casari, C. S.; Milani, A. Carbyne: From the Elusive Allotrope to Stable Carbon Atom Wires. MRS Commun. 2018, 8, 207-219.

(16) Zhao, X.; Ando, Y.; Liu, Y.; Jinno, M.; Suzuki, T. Carbon Nanowire Made of a Long Linear Carbon Chain Inserted inside a Multiwalled Carbon Nanotube. Phys. Rev. Lett. 2003, 90, 187401.

(17) Fantini, C.; Cruz, E.; Jorio, A.; Terrones, M.; Terrones, H.; Van Lier, G.; Charlier, J.-C.; Dresselhaus, M. S.; Saito, R.; Kim, Y. A.; Hayashi, T.; Muramatsu, H.; Endo, M.; Pimenta, M. A. Resonance Raman Study of Linear Carbon Chains Formed by the Heat Treatment of Double-Wall Carbon Nanotubes. Phys. Rev. B: Condens. Matter Mater. Phys. 2006, 73, 193408.

(18) Shi, L.; Sheng, L.; Yu, L.; An, K.; Ando, Y.; Zhao, X. Ultra-Thin Double-Walled Carbon Nanotubes: A Novel Nanocontainer for Preparing Atomic Wires. Nano Res. 2011, 4, 759-766.

(19) Andrade, N. F.; Vasconcelos, T. L.; Gouvea, C. P.; Archanjo, B. S.; Achete, C. A.; Kim, Y. A.; Endo, M.; Fantini, C.; Dresselhaus, M. S.; Souza Filho, A. G. Linear Carbon Chains Encapsulated in Multiwall Carbon Nanotubes: Resonance Raman Spectroscopy and Transmission Electron Microscopy Studies. Carbon 2015, 90, 172180.

(20) Shi, L.; Rohringer, P.; Suenaga, K.; Niimi, Y.; Kotakoski, J.; Meyer, J. C.; Peterlik, H.; Wanko, M.; Cahangirov, S.; Rubio, A.; Lapin, Z. J.; Novotny, L.; Ayala, P.; Pichler, T. Confined Linear Carbon Chains as a Route to Bulk Carbyne. Nat. Mater. 2016, 15, 634-639.

(21) Zhang, Y.; Zhao, J.; Fang, Y.; Liu, Y.; Zhao, X. Preparation of Long Linear Carbon Chain inside Multi-Walled Carbon Nanotubes by Cooling Enhanced Hydrogen Arc Discharge Method. Nanoscale 2018, 10, 17824-17833.

(22) Toma, S.; Asaka, K.; Irita, M.; Saito, Y. Bulk Synthesis of Linear Carbon Chains Confined inside Single-Wall Carbon Nanotubes by Vacuum Discharge. Surf. Interface Anal. 2019, 51, 131-135.

(23) Shi, L.; Senga, R.; Suenaga, K.; Kataura, H.; Saito, T.; Paz, A. P.; Rubio, A.; Ayala, P.; Pichler, T. Toward Confined Carbyne with Tailored Properties. Nano Lett. 2021, 21, 1096.

(24) Thomsen, C.; Reich, S. In Light Scattering in Solids IX: Novel Materials and Techniques; Cardona, M., Merlin, R., Eds.; Top. Appl. Phys.; Springer-Verlag Berlin Heidelberg: Berlin, Heidelberg, 2007; Vol. 108; pp 115-232.

(25) Jorio, A.; Dresselhaus, M. S.; Saito, R.; Dresselhaus, G. Raman Spectroscopy in Graphene Related Systems; John Wiley \& Sons: Weinheim, 2011.

(26) Saito, R.; Tatsumi, Y.; Huang, S.; Ling, X.; Dresselhaus, M. S. Raman Spectroscopy of Transition Metal Dichalcogenides. J. Phys.: Condens. Matter 2016, 28, 353002.

(27) Cong, X.; Liu, X.-L.; Lin, M.-L.; Tan, P.-H. Application of Raman Spectroscopy to Probe Fundamental Properties of TwoDimensional Materials. npj 2D Mater. Appl. 2020, 4, 13.

(28) Cardona, M. In Light Scattering in Solids II: Basic Concepts and Instrumentation; Cardona, M., Güntherodt, G., Eds.; Springer Berlin Heidelberg: Berlin, Heidelberg, 1982; pp 19-178.

(29) Brown, S. D. M.; Corio, P.; Marucci, A.; Dresselhaus, M. S.; Pimenta, M. A.; Kneipp, K. Anti-Stokes Raman Spectra of SingleWalled Carbon Nanotubes. Phys. Rev. B: Condens. Matter Mater. Phys. 2000, 61, R5137-R5140.

(30) Gordeev, G.; Jorio, A.; Kusch, P.; Vieira, B. G. M.; Flavel, B.; Krupke, R.; Barros, E. B.; Reich, S. Resonant Anti-Stokes Raman Scattering in Single-Walled Carbon Nanotubes. Phys. Rev. B: Condens. Matter Mater. Phys. 2017, 96, 245415.

(31) Maher, R. C.; Cohen, L. F.; Gallop, J. C.; Le Ru, E. C.; Etchegoin, P. G. Temperature-Dependent Anti-Stokes/Stokes Ratios under Surface-Enhanced Raman Scattering Conditions. J. Phys. Chem. B 2006, 110, 6797-6803.

(32) Oron-Carl, M.; Krupke, R. Raman Spectroscopic Evidence for Hot-Phonon Generation in Electrically Biased Carbon Nanotubes. Phys. Rev. Lett. 2008, 100, 127401.
(33) Jorio, A.; Kasperczyk, M.; Clark, N.; Neu, E.; Maletinsky, P.; Vijayaraghavan, A.; Novotny, L. Optical-Phonon Resonances with Saddle-Point Excitons in Twisted-Bilayer Graphene. Nano Lett. 2014, 14, 5687-5692.

(34) Tschannen, C. D.; Gordeev, G.; Reich, S.; Shi, L.; Pichler, T.; Frimmer, M.; Novotny, L.; Heeg, S. Raman Scattering Cross Section of Confined Carbyne. Nano Lett. 2020, 20, 6750-6755.

(35) Song, D.; Wang, F.; Dukovic, G.; Zheng, M.; Semke, E. D.; Brus, L. E.; Heinz, T. F. Direct Measurement of the Lifetime of Optical Phonons in Single-Walled Carbon Nanotubes. Phys. Rev. Lett. 2008, 100, 225503

(36) Souza Filho, A. G.; Chou, S. G.; Samsonidze, G. G.; Dresselhaus, G.; Dresselhaus, M. S.; An, L.; Liu, J.; Swan, A. K.; Ünlü, M. S.; Goldberg, B. B.; Jorio, A.; Grüneis, A.; Saito, R. Stokes and Anti-Stokes Raman Spectra of Small-Diameter Isolated Carbon Nanotubes. Phys. Rev. B: Condens. Matter Mater. Phys. 2004, 69, 115428.

(37) Vasconcelos, T. L.; Archanjo, B. S.; Oliveira, B. S.; Valaski, R.; Cordeiro, R. C.; Medeiros, H. G.; Rabelo, C.; Ribeiro, A.; Ercius, P.; Achete, C. A.; Jorio, A.; Cançado, L. G. Plasmon-Tunable Tip Pyramids: Monopole Nanoantennas for Near-Field Scanning Optical Microscopy. Adv. Opt. Mater. 2018, 6, 1800528.

(38) Oliveira, B. S.; Archanjo, B. S.; Valaski, R.; Achete, C. A.; Cançado, L. G.; Jorio, A.; Vasconcelos, T. L. Nanofabrication of Plasmon-Tunable Nanoantennas for Tip-Enhanced Raman Spectroscopy. J. Chem. Phys. 2020, 153, 114201.

(39) Miranda, H.; Rabelo, C.; Vasconcelos, T. L.; Cançado, L. G.; Jorio, A. Optical Properties of Plasmon-Tunable Tip Pyramids for Tip-Enhanced Raman Spectroscopy. Phys. Status Solidi RRL 2020, 14, 2000212.

(40) Liu, H.-N.; Cong, X.; Lin, M.-L.; Tan, P.-H. The Intrinsic Temperature-Dependent Raman Spectra of Graphite in the Temperature Range from $4 \mathrm{~K}$ to $1000 \mathrm{~K}$. Carbon 2019, 152, 451-458.

(41) Calizo, I.; Balandin, A. A.; Bao, W.; Miao, F.; Lau, C. N. Temperature Dependence of the Raman Spectra of Graphene and Graphene Multilayers. Nano Lett. 2007, 7, 2645-2649.

(42) Sahoo, S.; Gaur, A. P. S.; Ahmadi, M.; Guinel, M. J.-F.; Katiyar, R. S. Temperature-Dependent Raman Studies and Thermal Conductivity of Few-Layer MoS2. J. Phys. Chem. C 2013, 117, 9042-9047.

(43) Late, D. J.; Shirodkar, S. N.; Waghmare, U. V.; Dravid, V. P.; Rao, C. N. R. Thermal Expansion, Anharmonicity and TemperatureDependent Raman Spectra of Single- and Few-Layer MoSe2 and WSe2. ChemPhysChem 2014, 15, 1592-1598.

(44) Viana, B. C.; Alencar, R. S.; Vieira, A. G.; Carozo, V.; Souza Filho, A. G.; Wang, J.; da Luz-Lima, C.; Feng, S.; Perea-López, N.; Zhu, J.; Terrones, M. Temperature- and Power-Dependent Phonon Properties of Suspended Few Layers of Tungsten Diselenide. Vib. Spectrosc. 2020, 111, 103169.

(45) Livneh, T.; Sterer, E. Resonant Raman Scattering at Exciton States Tuned by Pressure and Temperature in $2 \mathrm{H}-\mathrm{MoS}_{2}$. Phys. Rev. B: Condens. Matter Mater. Phys. 2010, 81, 195209.

(46) Fan, J.-H.; Gao, P.; Zhang, A.-M.; Zhu, B.-R.; Zeng, H.-L.; Cui, X.-D.; He, R.; Zhang, Q.-M. Resonance Raman Scattering in Bulk 2HMX2 ( $\mathrm{M}=\mathrm{Mo}, \mathrm{W} ; \mathrm{X}=\mathrm{S}, \mathrm{Se})$ and Monolayer MoS2. J. Appl. Phys. 2014, 115, 053527 .

(47) Zobeiri, H.; Xu, S.; Yue, Y.; Zhang, Q.; Xie, Y.; Wang, X. Effect of Temperature on Raman Intensity of nm-Thick WS2: Combined Effects of Resonance Raman, Optical Properties, and Interface Optical Interference. Nanoscale 2020, 12, 6064-6078.

(48) Novotny, L.; Hecht, B. Principles of Nano-Optics; Cambridge University Press: Cambridge, 2012.

(49) Fantini, C.; Jorio, A.; Souza, M.; Strano, M. S.; Dresselhaus, M. S.; Pimenta, M. A. Optical Transition Energies for Carbon Canotubes from Resonant Raman Spectroscopy: Environment and Temperature Effects. Phys. Rev. Lett. 2004, 93, 147406.

(50) Parra-Murillo, C. A.; Santos, M. F.; Monken, C. H.; Jorio, A. Stokes-Anti-Stokes Correlation in the Inelastic Scattering of Light by 
Matter and Generalization of the Bose-Einstein Population Function. Phys. Rev. B: Condens. Matter Mater. Phys. 2016, 93, 125141.

(51) Zhou, Z.; Dou, X.; Ci, L.; Song, L.; Liu, D.; Gao, Y.; Wang, J.; Liu, L.; Zhou, W.; Xie, S.; Wan, D. Temperature Dependence of the Raman Spectra of Individual Carbon Nanotubes. J. Phys. Chem. B 2006, 110, 1206-1209.

(52) Zhang, Y.; Son, H.; Zhang, J.; Kong, J.; Liu, Z. Laser-Heating Effect on Raman Spectra of Individual Suspended Single-Walled Carbon Nanotubes. J. Phys. Chem. C 2007, 111, 1988-1992.

(53) Pozzi, E. A.; Zrimsek, A. B.; Lethiec, C. M.; Schatz, G. C.; Hersam, M. C.; Van Duyne, R. P. Evaluating Single-Molecule Stokes and Anti-Stokes SERS for Nanoscale Thermometry. J. Phys. Chem. C 2015, 119, 21116-21124.

(54) Bohn, J. E.; Etchegoin, P. G.; Le Ru, E. C.; Xiang, R.; Chiashi, S.; Maruyama, S. Estimating the Raman Cross Sections of Single Carbon Nanotubes. ACS Nano 2010, 4, 3466-3470.

(55) M, T.; Thripuranthaka, M.; Late, D. J. Temperature Dependent Phonon Shifts in Single-Layer WS2. ACS Appl. Mater. Interfaces 2014, 6, 1158-1163.

(56) Ferrari, A. C.; Basko, D. M. Raman Spectroscopy as a Versatile

Tool for Studying the Properties of Graphene. Nat. Nanotechnol. 2013, 8, 235-246.

(57) Roldán, R.; Castellanos-Gomez, A.; Cappelluti, E.; Guinea, F. Strain Engineering in Semiconducting Two-Dimensional Crystals. J. Phys.: Condens. Matter 2015, 27, 313201.

(58) Iqbal, M. W.; Shahzad, K.; Akbar, R.; Hussain, G. A Review on Raman Finger Prints of Doping and Strain Effect in TMDCs. Microelectron. Eng. 2020, 219, 111152.

(59) Wanko, M.; Cahangirov, S.; Shi, L.; Rohringer, P.; Lapin, Z. J.; Novotny, L.; Ayala, P.; Pichler, T.; Rubio, A. Polyyne Electronic and Vibrational Properties under Environmental Interactions. Phys. Rev. B: Condens. Matter Mater. Phys. 2016, 94, 195422.

(60) Balandin, A. A. Nanoscale Thermal Management. IEEE Potentials 2002, 21, 11-15.

(61) Cahill, D. G.; Ford, W. K.; Goodson, K. E.; Mahan, G. D.; Majumdar, A.; Maris, H. J.; Merlin, R.; Phillpot, S. R. Nanoscale Thermal Transport. J. Appl. Phys. 2003, 93, 793-818.

(62) van Erp, R.; Soleimanzadeh, R.; Nela, L.; Kampitsis, G.; Matioli, E. Co-Designing Electronics with Microfluidics for More Sustainable Cooling. Nature 2020, 585, 211-216.

(63) Shi, L.; Yanagi, K.; Cao, K.; Kaiser, U.; Ayala, P.; Pichler, T. Extraction of Linear Carbon Chains Unravels the Role of the Carbon Nanotube Host. ACS Nano 2018, 12, 8477-8484.

(64) Chimborazo, J.; Saito, T.; Pichler, T.; Shi, L.; Ayala, P. Towards Controllable Inner Chirality in Double-Walled Carbon Nanotubes. Appl. Phys. Lett. 2019, 115, 103102.

(65) Kamei, Y.; Suzuki, M.; Watanabe, K.; Fujimori, K.; Kawasaki, T.; Deguchi, T.; Yoneda, Y.; Todo, T.; Takagi, S.; Funatsu, T.; Yuba, S. Infrared Laser-Mediated Gene Induction in Targeted Single Cells in Vivo. Nat. Methods 2009, 6, 79-81.

(66) Xu, G.; Stevens, S. M., Jr.; Kobeissy, F.; Brown, H.; McClung, S.; Gold, M. S.; Borchelt, D. R. Identification of Proteins Sensitive to Thermal Stress in Human Neuroblastoma and Glioma Cell Lines. PLoS One 2012, 7, e49021.

(67) Kumar, S. V.; Wigge, P. A. H2A.Z-Containing Nucleosomes Mediate the Thermosensory Response in Arabidopsis. Cell 2010, 140, 136-147.

(68) Lemos, D.; Oliveira, T.; Martins, L.; de Azevedo, V. R; Rodrigues, M. F.; Ketzer, L. A.; Rumjanek, F. D. Isothermal Microcalorimetry of Tumor Cells: Enhanced Thermogenesis by Metastatic Cells. Front. Oncol. 2019, 9, 1430.

(69) Tran, H. N.; Blancon, J.-C.; Huntzinger, J.-R.; Arenal, R.; Popov, V. N.; Zahab, A. A.; Ayari, A.; San-Miguel, A.; Vallée, F.; Del Fatti, N.; Sauvajol, J.-L.; Paillet, M. Excitonic Optical Transitions Characterized by Raman Excitation Profiles in Single-Walled Carbon Nanotubes. Phys. Rev. B: Condens. Matter Mater. Phys. 2016, 94, 075430 .
(70) Hartschuh, A.; Sánchez, E. J.; Xie, X. S.; Novotny, L. HighResolution Near-Field Raman Microscopy of Single-Walled Carbon Nanotubes. Phys. Rev. Lett. 2003, 90, 095503.

(71) Lapin, Z. J.; Beams, R.; Cançado, L. G.; Novotny, L. Near-Field Raman Spectroscopy of Nanocarbon Materials. Faraday Discuss. 2015, 184, 193-206. 